\title{
Demethylzeylasteral ameliorates inflammation in a rat model of unilateral ureteral obstruction through inhibiting activation of the NF-кB pathway
}

\author{
QIANG WANG $^{1 *}$, YANMEI XIAO ${ }^{2 *}$, TONGWEI LIU ${ }^{3}$, HAIBO YUAN ${ }^{3}$ and CHUNWU LI $^{3}$ \\ ${ }^{1}$ Department of Urology, The First Central Hospital of Baoding; Departments of ${ }^{2}$ Nephrology \\ and ${ }^{3}$ Urology, The 252nd Hospital of The PLA, Baoding, Hebei 071000, P.R. China
}

Received February 2, 2016; Accepted January 23, 2017

DOI: $10.3892 / \mathrm{mmr} .2017 .6584$

\begin{abstract}
The present study investigated the pharmacodynamic role and therapeutic mechanism of demethylzeylasteral in the suppression of inflammation in a rat model of unilateral ureteral obstruction and reduction in nuclear factor (NF)- $\mathrm{\kappa B}$ pathway activity. The rats in the unilateral ureteral obstruction model were treated with $30-120 \mathrm{mg} / \mathrm{kg}$ demethylzeylasteral for 8 weeks. The activities of tumor necrosis factor (TNF)- $\alpha$, interleukin (IL)-6 and caspase-3/9, and the protein expression levels of cyclooxygenase (COX)-2 and intercellular adhesion molecule-1 (ICAM-1) and NF- $\mathrm{B}$ p 65 were analyzed using ELISA kits and western blot analyses, respectively. Compared with the rats in the unilateral ureteral obstruction model group, demethylzeylasteral treatment markedly inhibited the increased concentrations of serum creatinine and blood urea nitrogen, urinary protein/creatinine ratio, and concentrations of high-density lipoprotein and low-density lipoprotein cholesterol, and prevented kidney damage. In addition, demethylzeylasteral inhibited the levels of TNF- $\alpha$ andIL-6 and suppressed the protein expression levels of COX-2 and ICAM-1 in the kidneys of the rats in the unilateral ureteral obstruction model. Demethylzeylasteral also significantly suppressed the protein expression of NF- $\mathrm{KB}$ p65. The results of the present study suggested that demethylzeylasteral unilateral ureteral obstruction and inhibited inflammation via inhibiting the activation of COX-2, ICAM-1 and NF- $\mathrm{KB}$ p65, and suppressing the activities of caspase-3/9 in rats with unilateral ureteral obstruction.
\end{abstract}

Correspondence to: Dr Qiang Wang, Department of Urology, The First Central Hospital of Baoding, 320 The Great Wall, Baoding, Hebei 071000, P.R. China

E-mail: qiangwangqiang@yeah.net

*Contributed equally

Key words: demethylzeylasteral, unilateral ureteral obstruction, inflammation, nuclear factor- $\kappa \mathrm{B}$

\section{Introduction}

The morbidity rates of chronic kidney disease (CKD) are increasing yearly, however, no effective therapeutic methods have been found (1). Consequently, CKD has become a public health issue, which seriously threatens the health of individuals. Previous studies have predicted that $23-36 \%$ of the worldwide population suffer from CKD $(1,2)$. In central China, $\sim 10.49 \%$ of the population suffer from at least one type of disease resulting in renal injury (3). CKD has been generally considered to be closely associated with mortality rates in the young. It is characterized by high rates of mortality, morbidity and cardiovascular disease, whereas rates of control and awareness are low and have various complications. It has been suggested that, regardless of the cause of renal function injury, it is significantly associated with renal fibrotic lesions (2).

At present, as an experimental model can be readily and conveniently established through rat unilateral urethral obstruction, it is used extensively in renal interstitial fibrosis assessments (4). In addition, following model establishment, higher survival rates and a lower incidence of complications occur in the stable model, which can effectively reduce costs and experimental durations (5). A literature review found that unilateral urethral obstruction models have been used in the majority of studies on renal interstitial fibrosis and conclusions have been recognized generally (6). This is sufficient to confirm that this model is stable and has widespread approval from scholars (7).

As an important factor for the control of genetic transcription, nuclear factor (NF) $-\kappa B$ may be activated by the stimulation of cytokines, protein kinase, viruses and oxidizing agents (8). The activated NF- $\mathrm{KB}$ can induce the expressions of cytokines, growth factors and chemotactic factors, and be involved in pathological processes, including inflammatory responses, fibrosis, tumor development and atherosclerosis (9). Activated NF- $\mathrm{KB}$ can promote the transcription of monocyte chemoattractant protein-1, the excessive expression of which reduces the infiltration of macrophages and interstitial fibrosis $(10,11)$.

Demethylzeylasteral (molecular weight, 480; molecular formula, $\mathrm{C}_{29} \mathrm{H}_{36} \mathrm{O}_{6}$ ) is extracted from Tripterygium wilfordii (12). It can decrease the receptor activities of 
interleukin (IL)-2 through inhibition of the transformation of $\mathrm{T}$ cells, and the formation and release of IL-2 (13). Therefore, demethylzeylasteral may be a drug with high immunosuppressive and low myelosuppressive activities. In addition, according to assessments of the acute and subactute toxicity of demethylzeylasteral, it has not been found to cause renal toxicity (14). In the present study, it was hypothesized that demethylzeylasteral ameliorates inflammation in a rat model of unilateral ureteral obstruction, which was investigated, and it was demonstrated to suppress the NF- $\mathrm{kB}$ pathway were investigated.

\section{Materials and methods}

Animals and establishment of the model of unilateral ureteral obstruction. Specific pathogen-free male Sprague-Dawley rats weighing 220-250 g were purchased from Hebei University Laboratory Animal Center (Hebei, China) and were maintained with a standard laboratory diet under controlled indoor temperature $\left(22 \pm 1^{\circ} \mathrm{C}\right)$ and humidity $(65-70 \%)$ conditions. The animal experiments were approved by the Institute of The 252nd Hospital of the PLA (Baoding, China). All male Sprague-Dawley rats were randomly and equally divided into five groups (A-E; $\mathrm{n}=10$ rats/group) as follows: Group A, control group; Group B, model group; Group C, $30 \mathrm{mg} / \mathrm{kg}$ demethylzeylasteral group (Aburaihan Pharmaceutical Company, Tehran, Iran; Fig. 1); Group D, 60 mg/kg demethylzeylasteral group; Group E, $120 \mathrm{mg} / \mathrm{kg}$ demethylzeylasteral group. The male Sprague-Dawley rats were anesthetized with $2 \%$ pelltobarbitalum natricum $(40 \mathrm{mg} / \mathrm{kg})$. Under anesthesia, the left ureter of each rat was exposed and separated through a flank incision. The left ureter was then ligated with 4-0 silk, severed between the ligatures and left undisturbed. Following 8 weeks of treatment with demethylzeylasteral, kidney samples and blood were collected, fixed in $4 \%$ neutral-buffered formalin and stored at $-80^{\circ} \mathrm{C}$.

Laboratory biochemical analysis. Following 8 weeks of treatment with demethylzeylasteral, urine and serum samples from the Sprague-Dawley rats were collected and analyzed at The 252nd Hospital of the PLA. The concentrations of urinary protein and serum creatinine, and blood urea nitrogen, the urinary protein/creatinine $(\mathrm{Up} / \mathrm{Ucr})$ ratio, and serum high-density lipoprotein (HDL)-cholesterol (C) and low-density lipoprotein (LDL)-C concentrations were measured.

Histopathological examinations. Following 8 weeks of treatment with demethylzeylasteral, the Sprague-Dawley rats were sacrificed with excess pelltobarbitalum natricum. The kidney samples were collected and fixed in $4 \%$ neutral-buffered formalin. Subsequently, the paraffin sections $(4 \mu \mathrm{M})$ were stained with hematoxylin and eosin to reveal histopathological lesions which were visualized using a Zeiss Axioskop 40 microscope (Carl Zeiss, Oberkochen, Germany). Kidney samples were evaluated using a semi-quantitative scale from 0 to 3: Score 0 , absent; score 1, mild; score 2, moderate; score 3, severe.

Analyses of TNF- $\alpha$, IL-6, caspase-3/9 activities using ELISA. Following 8 weeks of treatment with demethylzeylasteral, the

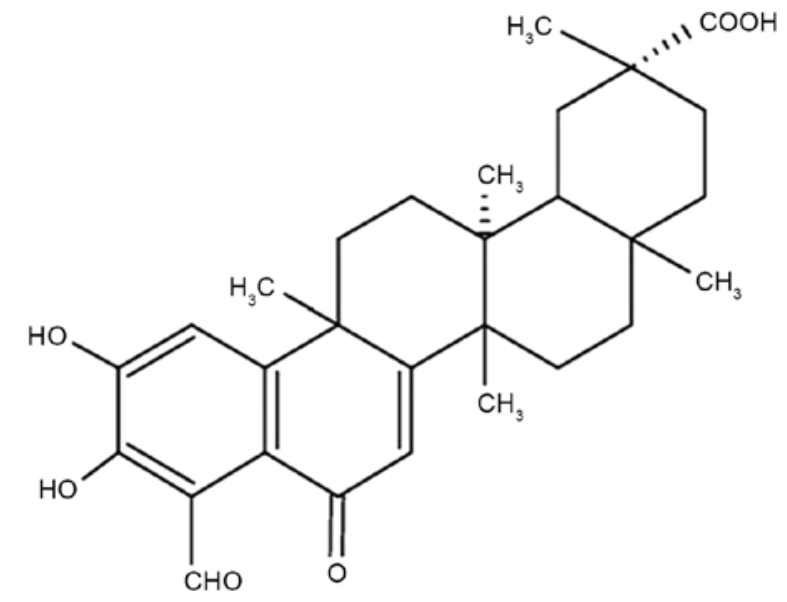

Figure 1. Chemical structure of demethylzeylasteral.

Sprague-Dawley rats were sacrificed with excess pelltobarbitalum natricum. Blood samples $(500 \mu \mathrm{l})$ were collected and centrifuged at $10,000 \mathrm{xg}$ for $10 \mathrm{~min}$ at $4^{\circ} \mathrm{C}$. The serum was then collected to analyze the activities of TNF- $\alpha$, IL- 6 and caspase-3/9 using ELISA kits.

Western blot analysis. Following 8 weeks of treatment with demethylzeylasteral, the Sprague-Dawley rats were sacrificed with excess pelltobarbitalum natricum. Kidney tissue samples were collected and lysed in RIPA buffer. Proteins were quantified using a BCA assay according to the manufacturer's protocol (Roche Diagnostics, Basel, Switzerland). The proteins $(50 \mu \mathrm{g})$ were electrophoresed on a 10\% polyacrylamide gel containing SDS and transferred onto nitrocellulose membranes (EMD Millipore, Bedford, MA, USA). The membranes were blocked with TBS- $0.05 \%$ Tween-20 (TBST) containing 5\% non-fat milk for $2 \mathrm{~h}$ at room temperature, and then incubated with anti-cyclooxygenase-2 (COX-2; 1:3,000; cat. no. 12282; Cell Signaling Technology, Inc., Boston, MA, USA), anti-intercellular adhesion molecule-1 (ICAM-1; 1:3,000; cat. no. ab20; Abcam, Cambridge, UK), anti-NF-кB p65 (1:3,000; cat. no. 8242; Cell Signaling Technology, Inc.) and $\beta$-actin $(1: 1,000$; cat. no. 4970; Cell Signaling Technology, Inc.) overnight at $4^{\circ} \mathrm{C}$. The membranes were washed with TBST and then incubated with mouse anti-rabbit IgG-HRP (1:5,000; cat. no. sc-2357; Santa Cruz Biotechnology, Inc., Dallas, TX, USA) for $2 \mathrm{~h}$ at room temperature and observed using an enhanced ECL chemiluminescence system (Pierce; Thermo Fisher Scientific, Inc., Waltham, MA, USA). Experiments were repeated three times. Quantification was performed using Image-Pro Plus version 6.0 software (Media Cybernetics, Inc., Rockville, MD, USA).

Statistical analysis. Data are expressed as the mean \pm standard error of the mean. The significance of results obtained from the control and treated groups was determined by one-way analysis of the variance followed by the Newman-Keuls test for multiple comparisons using SPSS version 20.0 software (IBM SPSS, Armonk, NY, USA). $\mathrm{P}<0.05$ was considered to indicate a statistically significant difference. 


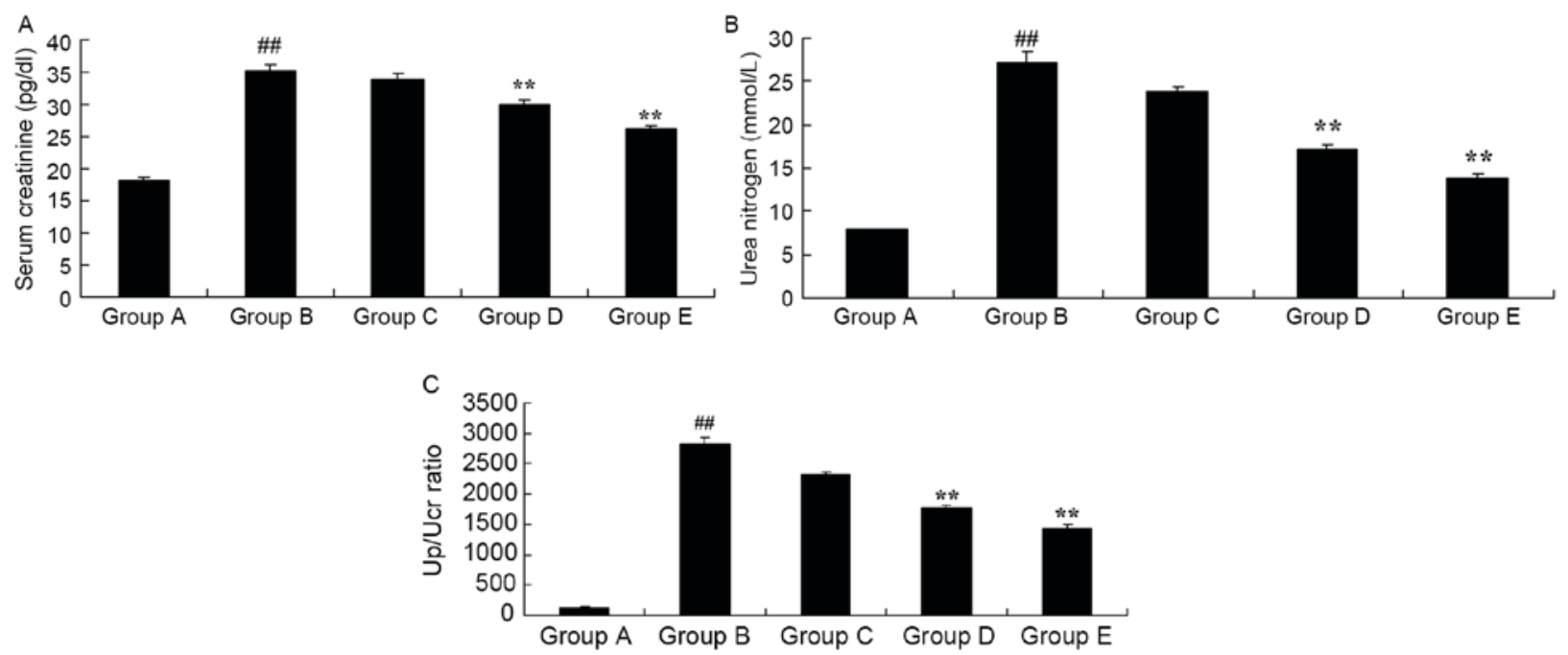

Figure 2. Demethylzeylasteral ameliorates biochemical indicators in urine/serum in a rat model of unilateral ureteral obstruction. Demethylzeylasteral ameliorated levels of (A) serum creatinine and (B) blood urea nitrogen, and (C) decreased the Up/Ucr ratio in the rat model of unilateral ureteral obstruction. Group A,

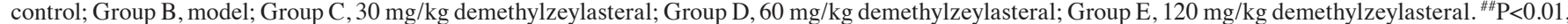
compared with Group A; ${ }^{* *} \mathrm{P}<0.01$, compared with Group B. Up/Ucr, urinary protein/creatinine.
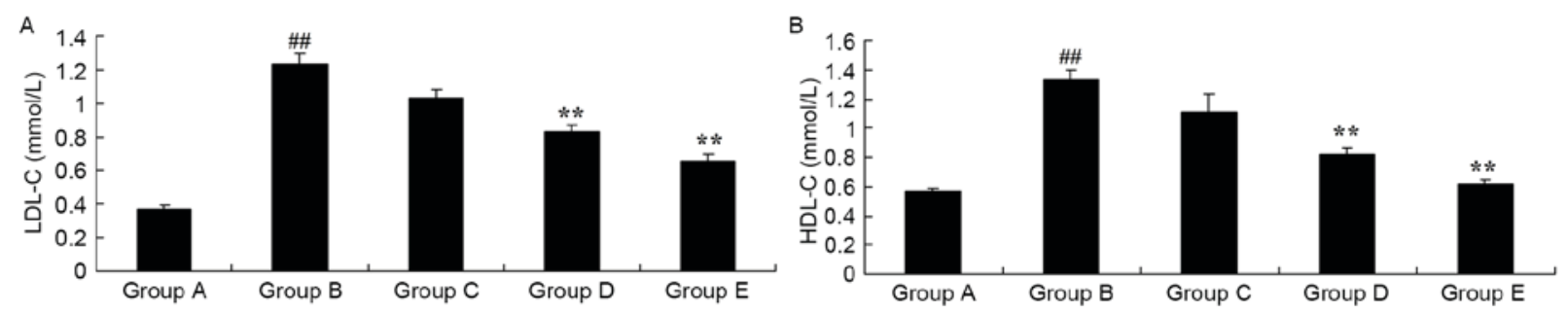

Figure 3. Demethylzeylasteral ameliorates HDL-C and LDL-C concentrations in a rat model of unilateral ureteral obstruction. Demethylzeylasteral ameliorated the concentrations of (A) LDL-C and (B) HDL-C in the rat model of unilateral ureteral obstruction. Group A, control; Group B, model; Group C, $30 \mathrm{mg} / \mathrm{kg}$ demethylzeylasteral; Group D, $60 \mathrm{mg} / \mathrm{kg}$ demethylzeylasteral; Group E, $120 \mathrm{mg} / \mathrm{kg}$ demethylzeylasteral. ${ }^{\# \#} \mathrm{P}<0.01$, compared with Group A; ${ }^{* * *} \mathrm{P}<0.01$, compared with Group B. HDL-C, high-density lipoprotein cholesterol; LDL-C, low-density lipoprotein cholesterol.

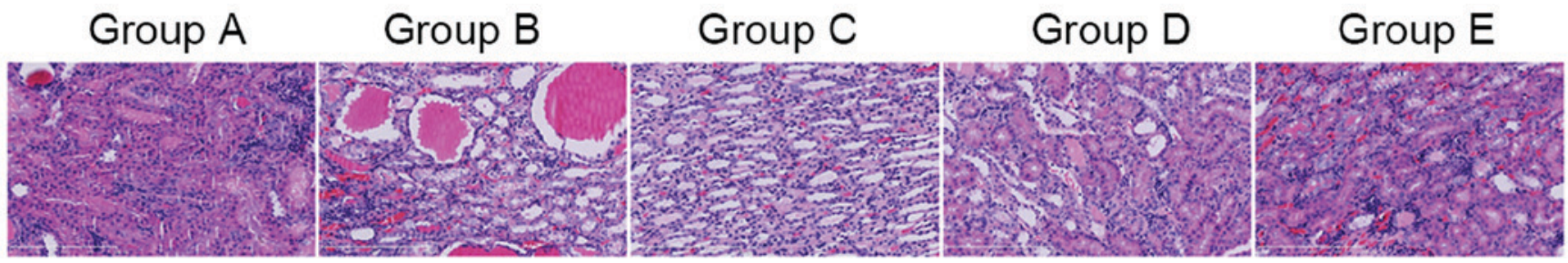

Figure 4. Demethylzeylasteral ameliorates kidney damage in a rat model of unilateral ureteral obstruction (magnification, x40). Group A, control; Group B, model; Group C, 30 mg/kg demethylzeylasteral; Group D, 60 mg/kg demethylzeylasteral; Group E, 120 mg/kg demethylzeylasteral.

\section{Results}

Demethylzeylasteral ameliorates the biochemical indicators of urine/serum in a rat model of unilateral ureteral obstruction. The chemical structure of demethylzeylasteral is shown in Fig. 1. Following 8 weeks of treatment with demethylzeylasteral, the levels of serum creatinine, blood urea nitrogen and the Up/Ucr ratio were markedly higher, compared with those in the normal control group (Fig. 2). Demethylzeylasteral treatment (60 or $120 \mathrm{mg} / \mathrm{kg}$ ) markedly inhibited the increases in serum creatinine, blood urea nitrogen and $\mathrm{Up} / \mathrm{Ucr}$ ratio in the rat model of unilateral ureteral obstruction (Fig. 2A-C).
Demethylzeylasteral ameliorates $H D L-C$ and $L D L-C$ concentrations in a rat model of unilateral ureteral obstruction. Following 8 weeks of treatment with demethylzeylasteral, the concentrations of HDL-C and LDL-C were examined in the rat model of unilateral ureteral obstruction to evaluate the effect of demethylzeylasteral on unilateral ureteral obstruction. The concentrations of HDL-C and LDL-C were also markedly higher, compared with those in the control normal rat (Fig. 3A and B). Treatment with 60 or $120 \mathrm{mg} / \mathrm{kg}$ of demethylzeylasteral significantly inhibited HDL-C and LDL-C concentrations in the rat model of unilateral ureteral obstruction (Fig. 3). 

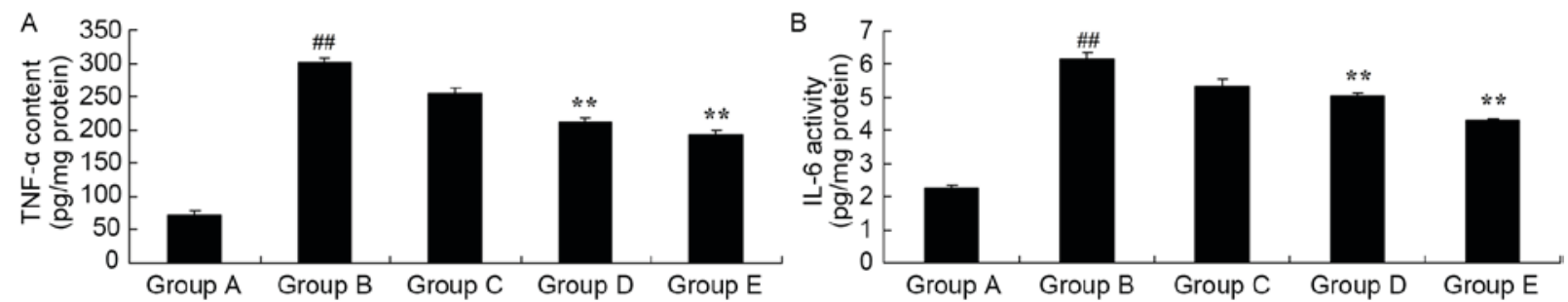

Figure 5. Demethylzeylasteral ameliorates TNF- $\alpha$ and IL-6 activities in a rat model of unilateral ureteral obstruction. Demethylzeylasteral ameliorated the activities of (A) TNF- $\alpha$ and (B) IL-6 in the rat model of unilateral ureteral obstruction. Group A, control; Group B, model; Group C, $30 \mathrm{mg} / \mathrm{kg}$ demethylzeylasteral; Group D, $60 \mathrm{mg} / \mathrm{kg}$ demethylzeylasteral; Group E, $120 \mathrm{mg} / \mathrm{kg}$ demethylzeylasteral. ${ }^{\# \#} \mathrm{P}<0.01$, compared with Group A; ${ }^{* *} \mathrm{P}<0.01$, compared with Group B. TNF- $\alpha$, tumor necrosis factor- $\alpha$; IL-6, interleukin- 6 .
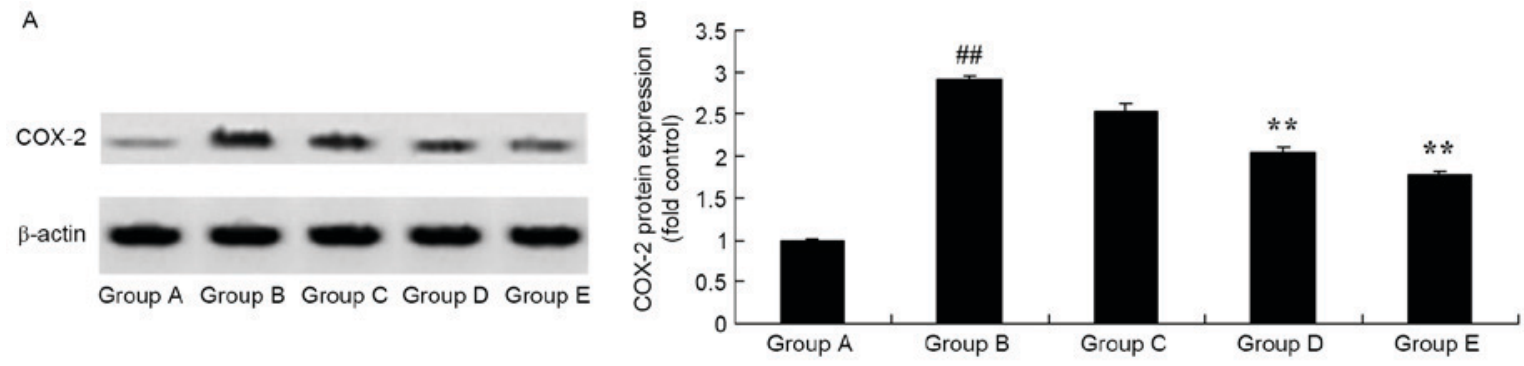

Figure 6. Demethylzeylasteral ameliorates the protein expression of COX-2 in a rat model of unilateral ureteral obstruction. (A) Demethylzeylasteral ameliorated the protein expression of COX-2, determined using western blot analysis. (B) Statistical analysis of the protein expression of COX-2 in the rat model of unilateral ureteral obstruction. Group A, control; Group B, model; Group C, $30 \mathrm{mg} / \mathrm{kg}$ demethylzeylasteral; Group D, $60 \mathrm{mg} / \mathrm{kg}$ demethylzeylasteral; Group E, $120 \mathrm{mg} / \mathrm{kg}$ demethylzeylasteral. ${ }^{\# /} \mathrm{P}<0.01$, compared with Group A; ${ }^{* *} \mathrm{P}<0.01$, compared with Group B. COX-2, cyclooxygenase-2.
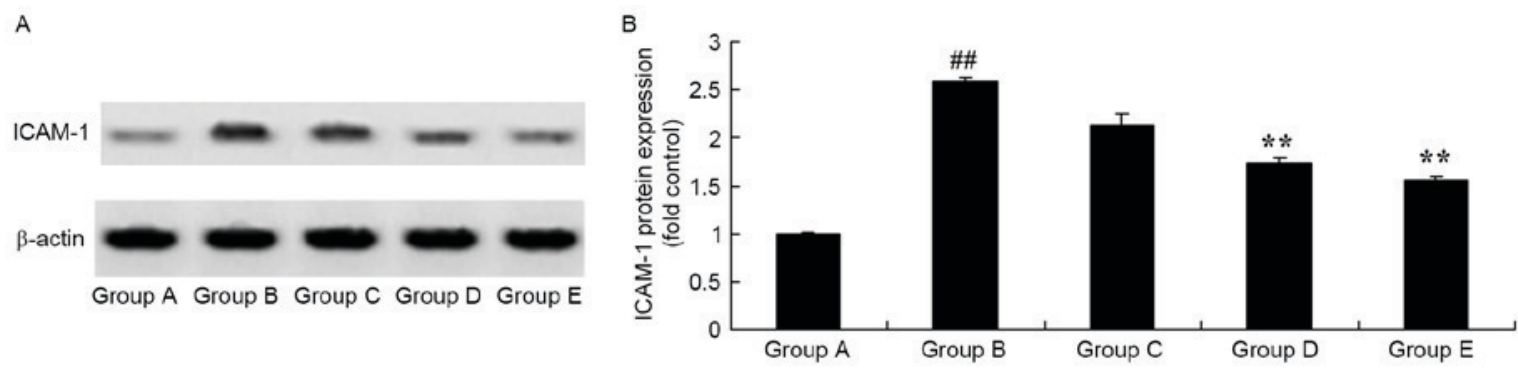

Figure 7. Demethylzeylasteral ameliorates the protein expression of ICAM-1 in a rat model of unilateral ureteral obstruction. (A) Western blot analysis revealed that demethylzeylasteral ameliorated the protein expression of ICAM-1. (B) Statistical analysis of the protein expression of ICAM-1 in the rat model of unilateral ureteral obstruction. Group A, control; Group B, model; Group C, $30 \mathrm{mg} / \mathrm{kg}$ demethylzeylasteral; Group D, $60 \mathrm{mg} / \mathrm{kg}$ demethylzeylasteral; Group E, $120 \mathrm{mg} / \mathrm{kg}$ demethylzeylasteral. ${ }^{\# /} \mathrm{P}<0.01$, compared with Group A; ${ }^{* *} \mathrm{P}<0.01$, compared with Group B. ICAM-1, intercellular adhesion molecule-1.

Demethylzeylasteral ameliorates kidney damage in a rat model of unilateral ureteral obstruction. Following 8 weeks of treatment, histopathological examinations were performed to evaluate the protective effect of demethylzeylasteral on kidney damage in the rat model of unilateral ureteral obstruction. The kidney damage score of the unilateral ureteral obstruction model group was higher, compared with that of the control group (Fig. 4). Treatment with 60 or $120 \mathrm{mg} / \mathrm{kg}$ demethylzeylasteral significantly prevented kidney damage in the rat model of unilateral ureteral obstruction (Fig. 4).

Demethylzeylasteral ameliorates TNF- $\alpha$ and IL- 6 activities in a rat model of unilateral ureteral obstruction. To investigate whether demethylzeylasteral has an anti-inflammatory effect in a rat model of unilateral ureteral obstruction, the present study examined the activities of TNF- $\alpha$ and IL- 6 using ELIST kits. As shown in Fig. 5, there were significant increases in the activities of TNF- $\alpha$ and IL-6 in the unilateral ureteral obstruction model, compared with activities in the normal control. Pretreatment with 60 or $120 \mathrm{mg} / \mathrm{kg}$ demethylzeylasteral significantly reduced the activities of TNF- $\alpha$ and IL- 6 in the unilateral ureteral obstruction group (Fig. 5A and B).

Demethylzeylasteral ameliorates the protein expression of COX-2 in a rat model of unilateral ureteral obstruction. To assess the protein expression of COX-2 in the protective effect of demethylzeylasteral on unilateral ureteral obstruction, the protein expression of COX-2 was examined using western blot analysis. The results of the western blot assay showed that the protein expression of COX-2 in the rats of the unilateral ureteral obstruction group was higher, compared with that in the rats in control group (Fig. 6A and B). However, 60 
A

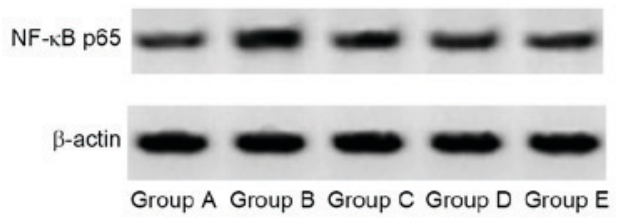

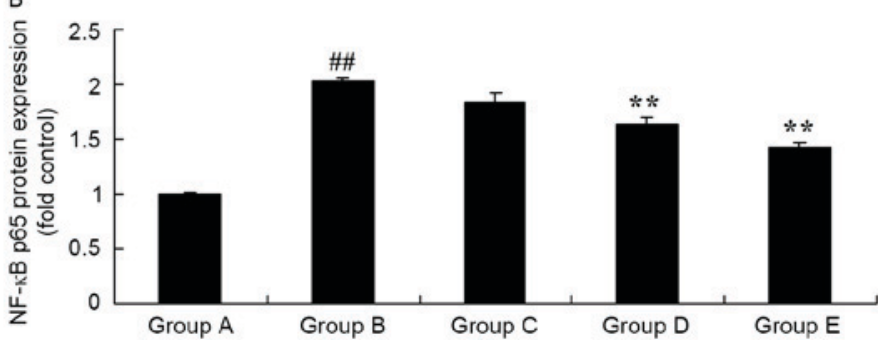

Figure 8. Demethylzeylasteral ameliorates the protein expression of NF-kB p65 in a rat model of unilateral ureteral obstruction. (A) Western blot analysis

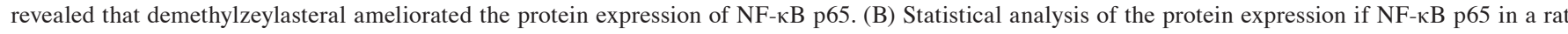
model of unilateral ureteral obstruction. Group A, control; Group B, model; Group C, 30 mg/kg demethylzeylasteral; Group D, $60 \mathrm{mg} / \mathrm{kg}$ demethylzeylasteral; Group E, $120 \mathrm{mg} / \mathrm{kg}$ demethylzeylasteral. ${ }^{\# \#} \mathrm{P}<0.01$, compared with Group $\mathrm{A} ;{ }^{* *} \mathrm{P}<0.01$, compared with Group B. NF- $\mathrm{kB}$, nuclear factor- $\mathrm{kB}$.
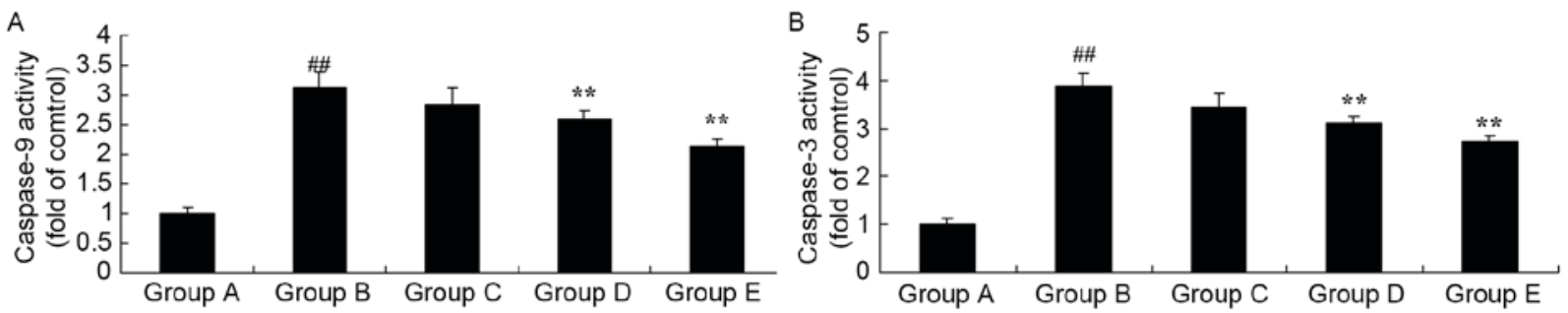

Figure 9. Demethylzeylasteral ameliorates the activities of caspase-3/9 in a rat model of unilateral ureteral obstruction. Demethylzeylasteral ameliorated the activities of (A) caspase-9 and (B) caspase-3 in the rat model of unilateral ureteral obstruction. Group A, control; Group B, model; Group C, $30 \mathrm{mg} / \mathrm{kg}$ demethylzeylasteral; Group D, $60 \mathrm{mg} / \mathrm{kg}$ demethylzeylasteral; Group E, $120 \mathrm{mg} / \mathrm{kg}$ demethylzeylasteral. ${ }^{\# \#} \mathrm{P}<0.01$, compared with Group A; ${ }^{* *} \mathrm{P}<0.01$, compared with Group B.

or $120 \mathrm{mg} / \mathrm{kg}$ demethylzeylasteral treatment significantly suppressed the protein expression of COX-2 in the unilateral ureteral obstruction group (Fig. 6A and B).

Demethylzeylasteral ameliorates the protein expression of ICAM-1 in a rat model of unilateral ureteral obstruction. To investigate whether ICAM-1 is involved in the protective effect of demethylzeylasteral on unilateral ureteral obstruction; western blot analysis was used to analyze the protein expression of ICAM-1. As shown in Fig. 7A and B, an increase in the protein expression of ICAM-1 was observed in the unilateral ureteral obstruction rats, compared with the normal rats. Treatment with 60 or $120 \mathrm{mg} / \mathrm{kg}$ demethylzeylasteral significantly reduced the protein expression of ICAM-1 in the unilateral ureteral obstruction model (Fig. 7).

Demethylzeylasteral ameliorates the protein expression of $N F-\kappa B$ p65 in a rat model of unilateral ureteral obstruction. To elucidate the mechanism underlying the anti-inflammatory effect of demethylzeylasteral in unilateral ureteral obstruction, the protein expression of NF- $\mathrm{KB}$ p 65 was examined using western blot analysis. Unilateral ureteral obstruction

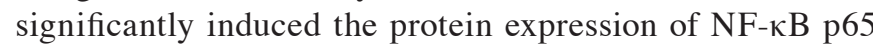
in the unilateral ureteral obstruction rats (Fig. 8A and B). Treatment with 60 or $120 \mathrm{mg} / \mathrm{kg}$ of demethylzeylasteral significantly inhibited the protein expression of NF- $\mathrm{KB}$ p65 in the rat model of unilateral ureteral obstruction (Fig. 8).

Demethylzeylasteral ameliorates the activities of caspase-3/9 in a rat model of unilateral ureteral obstruction. To further elucidate the mechanism of demethylzeylasteral on unilateral ureteral obstruction, cell apoptosis and caspase-3/9 activities were examined in the present study. The activities of caspase-3/9 were significantly increased in the unilateral ureteral obstruction model, compared with normal control group (Fig. 9A and B). Treatment with 60 or $120 \mathrm{mg} / \mathrm{kg}$ demethylzeylasteral significantly reduced the activities of caspase-3/9 in the unilateral ureteral obstruction rats (Fig. 9A and B).

\section{Discussion}

According to reliable data, the diagnosis of CKD based on the current method of assessment showed that, between 2009 and 2010, the morbidity rate in China was $10.8 \%$ and rates worldwide were $10-13 \%$ (3). Regardless of the causes of kidney disease, progressive CKD causes the formation of large scars, and finally result in the complete destruction of renal parenchyma and kidney failure at the terminal stage (15). In this state, renal dialysis or renal transplantation is required. CKD has become an extensive public health issue, which threatens the lives of individuals worldwide (16), and the substantial costs involved in prevention, diagnosis and treatment affect individuals worldwide. In the present study, it was found that demethylzeylasteral treatment markedly inhibited the increased concentrations of serum creatinine, blood urea nitrogen and the Up/Ucr ratio, inhibited the concentrations of HDL-C and LDL-C, prevented kidney damage, and reduced the activities of TNF- $\alpha$ and IL- 6 in a rat model of unilateral ureteral obstruction. These observations further supported the hypothesis that demethylzeylasteral alleviates unilateral ureteral obstruction through downstream pro-inflammatory mediators. 
Comprising the two isoenzymes of COX-1 and COX-2, COX is regarded as a key rate-limiting enzyme in the metabolic link of prostaglandin (17). COX-1 is involved in normal physiological processes whereas COX-2 can mediate pathological alterations (18). Studies have revealed that the proliferation of COX-2 is important in renal tubule interstitial fibrosis caused by ureteral obstruction, and overexpression of COX-2 in the renal cortex may be a promoting factor for inflammatory cell infiltration and accumulation $(19,20)$. In the present study, demethylzeylasteral treatment significantly suppressed the protein expression ofCOX-2 in the unilateral ureteral obstruction rats (14).

When renal inflammatory injury occurs, adhesion molecules mediate the adhesion of inflammatory cells on vascular walls and move to regions of inflammation, which promotes injuries (21). The continuous invasion of inflammatory cells is an important factor for the expansion of the renal inflammatory response and renal function injury. The two adhesion molecules, ICAM-1 in the immunogloblin superfamily and P-selectin in the selectin family $(21,22)$, were also examined. ICAM-1 is essential in early renal tubular interstitial fibrosis. P-selectin is present on the surface of activated endothelial cells and an early initiator of the inflammatory reaction (23). The present study found that demethylzeylasteral significantly reduced the protein expression of ICAM-1 in the unilateral ureteral obstruction rats. Hu et al (14) also suggested that demethylzeylasteral exhibits reno-protective effects through inhibiting the activation of COX-2 and ICAM-1.

NF- $\kappa B$ can form a tripolymer by combining with inhibitor of NF- $\mathrm{kB}$ (IkB). When IkB degrades and is lost, a dipolymer with transcriptional activity is formed (9). The activated dipolymer has the following functions in renal interstitial fibrosis (8): Firstly, it is associated with fibroblast growth factors generated by fibroblasts and intrinsic renal cells. Secondly, it is involved in forming chemokines and cell adhesion molecules. Thirdly, it facilitates the proliferation and division of fibroblasts (24). TNF can stimulate the separation of NF- $\mathrm{KB}$ with IKB and is involved in genetic regulation, inflammatory mediators and cytokines, including TNF (25). TNF primarily assembles inflammatory cells and integrates with receptors on the cell surface. Previous studies have found that, following unilateral urethral obstruction, the expression of NF- $\mathrm{\kappa B}$ in the renal interstitium is increased and the expression of renal interstitial collagen is upregulated (26). Peach kernels can also inhibit inflammatory mediators and associated cytokines, including TNF- $\alpha$ and transforming growth factor- $\beta$ (27). The present study demonstrated that demethylzeylasteral markedly inhibited the protein expression of NF-kB p65 and the activities of caspase-3/9 in a rat model of unilateral ureteral obstruction. Hu et al (14) also suggested that demethylzeylasteral exhibits reno-protective effects through inhibiting the activation of $\mathrm{NF}-\mathrm{\kappa B}$.

In conclusion, the present study demonstrated that demethylzeylasteral treatment markedly inhibited the increased serum creatinine, blood urea nitrogen and Up/Ucr ratio, and the concentrations of HDL-C and LDL-C. It also prevented kidney damage, and reduced the activities of TNF- $\alpha$ and IL- 6 in the rat model of unilateral ureteral obstruction. These effects were accompanied by inhibition of the activation of COX-2, ICAM-1 and NF- $\mathrm{kB}$ p65, and suppression of the activities of caspase-3/9 in the kidney of the rats with unilateral ureteral obstruction. Therefore, these results indicated that demethylzeylasteral may be a novel therapeutic agent for unilateral ureteral obstruction.

\section{References}

1. Bainey KR, Rahim S, Etherington K, Rokoss ML, Natarajan MK, Velianou JL, Brons S and Mehta SR; CAPTAIN Investigators: Effects of withdrawing vs continuing renin-angiotensin blockers on incidence of acute kidney injury in patients with renal insufficiency undergoing cardiac catheterization: Results from the angiotensin converting enzyme inhibitor/angiotensin receptor blocker and contrast induced nephropathy in patients receiving cardiac catheterization (CAPTAIN) trial. Am Heart J 170: 110-116, 2015.

2. Renke M, Lizakowski S, Tylicki L, Rutkowski P, Knap N, Heleniak Z, Sławińska-Morawska M, Aleksandrowicz-Wrona E, Januszczyk J, Wójcik-Stasiak M, et al: Aliskiren attenuates oxidative stress and improves tubular status in non-diabetic patients with chronic kidney disease-Placebo controlled, randomized, cross-over study. Adv Med Sci 59: 256-260, 2014.

3. Locatelli F, Mandolfo S, Menegato Adorati M, Villa G, Tarchini R, Pizzarelli F, Conte F, Guastoni C, Ricciardi B and Crotta A: Efficacy and safety of once-monthly continuous erythropoietin receptor activator in patients with chronic renal anemia. J Nephrol 26: 1114-1121, 2013.

4. Yasuda T, Endoh M, Suzuki D, Yoshimura A, Ideura T, Tamura K, Kamata K, Toya Y, Umemura S and Kimura K; KVT Study Group: Effects of valsartan on progression of kidney disease in Japanese hypertensive patients with advanced, predialysis, chronic kidney disease: Kanagawa Valsartan Trial (KVT). Hypertens Res 36: 240-246, 2013.

5. de Lusignan S, Tomson C, Harris K, van Vlymen J and Gallagher H: Creatinine fluctuation has a greater effect than the formula to estimate glomerular filtration rate on the prevalence of chronic kidney disease. Nephron Clin Pract 117: c213-c224, 2011.

6. Fang YQ, Qiu JG, Wang DJ, Zhan HL and Situ J: Comparative study on ureteroscopic lithotripsy and laparoscopic ureterolithotomy for treatment of unilateral upper ureteral stones. Acta Cir Bras 27: 266-270, 2012.

7. Patel M, Thimons DG, Winston JL, Langholff $\mathrm{W}$ and McGowan T: An open-label, randomized, multicenter, controlled study of epoetin alfa for the treatment of anemia of chronic kidney disease in the long term care setting. J Am Med Dir Assoc 13: 244-248, 2012.

8. Wang S, Liu K, Seneviratne CJ, Li X, Cheung GS, Jin L, Chu CH and Zhang C: Lipoteichoic acid from an clinical strain promotes TNF- $\alpha$ expression through the NF- $\kappa \mathrm{B}$ and p38 MAPK signaling pathways in differentiated THP-1 macrophages. Biomed Rep 3: 697-702, 2015.

9. Dhanasekar C, Kalaiselvan S and Rasool M: Morin, a bioflavonoid suppresses monosodium urate crystal-induced inflammatory immune response in RAW 264.7 macrophages through the inhibition of inflammatory mediators, intracellular ROS levels and NF-kappaB activation. PLoS One 10: e0145093, 2015.

10. Yang SY, Lin SL, Chen YM, Wu VC, Yang WS and Wu KD: Downregulation of angiotensin type 1 receptor and nuclear factor- $\mathrm{\kappa} \mathrm{B}$ by sirtuin 1 contributes to renoprotection in unilateral ureteral obstruction. Sci Rep 6: 33705, 2016.

11. Ozbek E, Ilbey YO, Ozbek M, Simsek A, Cekmen M and Somay A: Melatonin attenuates unilateral ureteral obstruction-induced renal injury by reducing oxidative stress, iNOS, MAPK, and NF-kB expression. J Endourol 23: 1165-1173, 2009.

12. Liu SL, Zhang SY, Wang MJ, Jiang H, Yang YX and Chen L: Demethylzeylasteral exhibits dose-dependent inhibitory behaviour towards estradiol glucuronidation. Eur J Drug Metab Pharmacokinet 39: 99-102, 2014.

13. Xu W, Lin Z, Yang C, Zhang Y, Wang G, Xu X, Lv Q, Ren Y and Dong Y: Immunosuppressive effects of demethylzeylasteral in a rat kidney transplantation model. Int Immunopharmacol 9: 996-1001, 2009.

14. Hu Q, Yang C, Wang Q, Zeng H and Qin W: Demethylzeylasteral (T-96) treatment ameliorates mice lupus nephritis accompanied by inhibiting activation of NF- $\mathrm{B}$ pathway. PLoS One 10: e0133724, 2015. 
15. Bellizzi V, Chiodini P, Cupisti A, Viola BF, Pezzotta M, De Nicola L, Minutolo R, Barsotti G, Piccoli GB and Di Iorio B: Very low-protein diet plus ketoacids in chronic kidney disease and risk of death during end-stage renal disease: A historical cohort controlled study. Nephrol Dial Transplant 30: 71-77, 2015.

16. Santana-Santos E, Gowdak LH, Gaiotto FA, Puig LB, Hajjar LA Zeferino SP, Drager LF, Shimizu MH, Bortolotto LA and De Lima JJ: High dose of $\mathrm{N}$-acetylcystein prevents acute kidney injury in chronic kidney disease patients undergoing myocardial revascularization. Ann Thorac Surg 97: 1617-1623, 2014.

17. Kuo FC, Tseng YT, Wu SR, Wu MT and Lo YC: Melamine activates NFKB/COX-2/PGE2 pathway and increases NADPH oxidase-dependent ROS production in macrophages and human embryonic kidney cells. Toxicol In Vitro 27: 1603-1611, 2013

18. Kim HS, Kim T, Kim MK, Suh DH, Chung HH and Song YS Cyclooxygenase-1 and -2: Molecular targets for cervical neoplasia. J Cancer Prev 18: 123-134, 2013.

19. Bautista-Garcia P, Sánchez-Lozada LG, Cristóbal-Garcia M, Tapia E, Soto V, Avila-Casado MC, Márquez-Velasco R, Bojalil R, Franco M and Herrera-Acosta J: Chronic inhibition of NOS-2 ameliorates renal injury, as well as COX-2 and TGF-beta 1 overexpression in 5/6 nephrectomized rats. Nephrol Dial Transplant 21: 3074-3081, 2006

20. Imig JD, Zhao X, Dey A and Shaw M: CYP450, COX-2 and obesity related renal damage. Toxicol Mech Methods 15 $125-136,2005$

21. Chen J, Hamm LL, Mohler ER, Hudaihed A, Arora R, Chen CS, Liu Y, Browne G, Mills KT, Kleinpeter MA, et al: Interrelationship of multiple endothelial dysfunction biomarkers with chronic kidney disease. PLoS One 10: e0132047, 2015.
22. Attalah MF, Adel LA and Fahmy DE: Diagnostic and prognostic values of adhesion molecules VCAM-1, ICAM-1 and C-reactive protein in Egyptian patients with chronic kidney disease. Egypt J Immunol 18: 59-66, 2011.

23. Vaccaro F, Mulè G, Cottone S, Soresi M, Giannitrapani L, Vadalà A, Sparacino V, Calabrese S, Picone FP, Montalto G and Cerasola G: Circulating levels of adhesion molecules in chronic kidney disease correlate with the stage of renal disease and with C-reactive protein. Arch Med Res 38: 534-538, 2007.

24. Shimizu H, Yisireyili M, Higashiyama Y, Nishijima F and Niwa T: Indoxyl sulfate upregulates renal expression of ICAM-1 via production of ROS and activation of NF- $\mathrm{BB}$ and $\mathrm{p} 53$ in proximal tubular cells. Life Sci 92: 143-148, 2013.

25. Percy CJ, Brown L, Power DA, Johnson DW and Gobe GC: Obesity and hypertension have differing oxidant handling molecular pathways in age-related chronic kidney disease. Mech Ageing Dev 130: 129-138, 2009.

26. Yu Y, Zhang L, Liu Q, Tang L, Sun H and Guo H: Endoplasmic reticulum stress preconditioning antagonizes low-density lipoprotein-induced inflammation in human mesangial cells through upregulation of XBP1 and suppression of the IRE1 $\alpha / \mathrm{IKK} / \mathrm{NF}-\kappa \mathrm{B}$ pathway. Mol Med Rep 11: 2048-2054, 2015.

27. Liu Z, Huang XR, Chen HY, Penninger JM and Lan HY: Loss of angiotensin-converting enzyme 2 enhances TGF- $\beta /$ Smad-mediated renal fibrosis and NF- $\mathrm{BB}$-driven renal inflammation in a mouse model of obstructive nephropathy. Lab Invest 92: 650-661, 2012. 